\title{
Condensate mechanism of conformal symmetry breaking
}

\author{
Victor Pervushin* \\ JINR \\ E-mail: pervush@theor.jinr.ru
}

Andrej Arbuzov, Boris Barbashov, Alexander Cherny, Alexander Dorokhov,

JINR, Dubna,

Andrzej Borowiec,

ITP, University of Wrocław, Poland,

Rashid Nazmitdinov,

JINR, Dubna and Department de Física, Universitat de les Illes Balears, Palma de Mal-

lorca, Spain,

Alexander Pavlov,

Moscow State Agri-Engineering University, 127550 Moscow, Russia,

Vadim Shilin,

JINR, Dubna and Moscow Institute of Physics and Technology, Dolgoprudny, Russia,

Alexander Zakharov,

ITEP, Moscow, Russia.

The low energy Gell-Mann-Oakes-Renner relation, the Higgs particle mass value, and the new observational cosmological data are considered as evidence of the condensate mechanism of conformal symmetry breaking at the quantum level. The condensate mechanism occurs by means of normal ordering of field operators in QCD, Minimal Standard Model of electroweak interactions without the Higgs potential, and the Dirac conformal General Relativity with long range forces.

XXI International Baldin Seminar on High Energy Physics Problems

September 10-15, 2012

JINR, Dubna, Russia

${ }^{*}$ Speaker. 


\section{Introduction}

The symmetry is inseparably connected with conception of beauty. At the same time, intrinsical beauty is achieved upon condition the symmetry has been breakdown a little and is incomplete. This incompleteness gives mysteriousness and attractiveness to the Nature.

In particular, in modern physics there is a tendency to treat the broken conformal symmetry as the basis of unification of all interactions. This is the reason to consider quantum chromodynamics (QCD), Minimal Standard Model (SM) of electroweak interactions, and the Dirac version of General Relativity (GR) with long range forces [1] in the framework of the conformal symmetry principle. Our approach to all these theories (QCD, SM, and GR) is based on their conformal invariant classical versions and the Dirac Hamiltonian scheme in a definite frame of reference [2]. It will be proposed that conformal symmetry can be broken at the quantum level by the normal ordering of field operators, which leads to their condensates and Casimir energies.

\section{Low Energy QCD}

The QCD description of hadrons is based on the non-Abelian generalization of the QED description of bound states $[3,4]$ in concordance with irreducible representations of the Poincaré group [5, 6], and the Dirac gauge invariant quantization [7], where the potential components are separated from radiation ones in a definite frame of reference.

Let us begin from the standard definition of Poincaré-invariant (P-inv) and gauge invariant (G-inv) S-matrix elements. Recall that the S-matrix elements are defined as the evolution operator expectation values between in- and out- states

$$
\underbrace{\mathscr{M}_{\text {in,out }}}_{P-\text { inv }, G-\text { inv }}=\underbrace{\langle\text { out }|}_{P-\text { variant }} \underbrace{\hat{S}[\hat{\ell}]}_{P-\text { variant }, G-\text { inv }} \underbrace{\mid \text { in }\rangle}_{P-\text { variant }} .
$$

The in- and out- states are the rays defined as the products of the P-variant representations [8]

$$
\langle\text { out }|=\left\langle\prod_{J} \mathscr{P}_{J}, s_{J}|, \quad| \text { in }\right\rangle=\left|\prod_{J} \mathscr{P}_{J}, s_{J}\right\rangle,
$$

where $\mathscr{P}_{J}, s_{J}$ are the total momentum and spin of a state $(J)$. This means that all particles (elementary and composite) are far enough from each other to neglect their interactions in the in- and outstates. These irreducible representations form a complete set of states. The frames of reference are distinguished by the eigenvalues of the appropriate time-like operator $\hat{\ell}_{\mu}=\frac{\hat{\mathscr{P}}_{\mu}}{M_{J}}$ (where $M_{J}$ is a particle mass). This operator acts in the complete set of these states:

$$
\hat{\ell}_{\mu}|\mathscr{P}, s\rangle=\frac{\mathscr{P}_{J \mu}}{M_{J}}\left|\mathscr{P}_{J}, s\right\rangle \text {. }
$$

The Bogoliubov-Logunov-Todorov rays (2.3) can include bound states [3, 4].

In a definite frame of reference the gauge invariance of the S-matrix elements (2.1) can be achieved via the Dirac Hamiltonian approach to QED of 1927. This approach was based on the constraint-shell action [7]

$$
W_{\mathrm{QED}}^{\mathrm{Dirac}}=W_{\mathrm{QED}} \mid \frac{\delta W_{\mathrm{QED}}}{\delta A_{0}^{\ell}}=0
$$


where the component $A_{0}^{\ell}$ is defined by the scalar product $A_{0}^{\ell}=A_{\mu} \ell^{\mu}=(A \cdot \ell)$ of the vector field $A_{\mu}$ and the unit time-like vector $\ell_{\mu}$ characterizing the frame of reference (2.3).

The gauge condition was established by Dirac as the first integral of the Gauss constraint

$$
\int^{t} d t \frac{\delta W_{\mathrm{QED}}}{\delta A_{0}^{\ell}}=0, \quad t=(x \cdot \ell) .
$$

In this case, the S-matrix elements (2.1) are relativistic invariant and independent of the frame reference provided the condition (2.3) is fulfilled [9].

The generalization of the Dirac Hamiltonian approach to QED of 1927 to any frame was discussed by Heisenberg and Pauli's in 1930 [10]. It was their question to von Neumann who suggested them to go back to the initial Lorentz-invariant formulation and choose the comoving frame and repeat the gauge invariant Dirac scheme in this frame. The comoving frame time axis $\ell_{\mu}$ (2.3) for a bound state is proportional to the total momentum operator [5, 6].

The next concept of our scheme is the normal ordering of field operators in this comoving frame. It is well known that the normal ordering of the oscillator Hamiltonian

$$
: \sum_{n} \frac{p_{n}^{2}+\omega_{n}^{2} q_{n}^{2}}{2}:=: \sum_{n} \omega_{n} \frac{a_{n}^{+} a_{n}^{-}+a_{n}^{-} a_{n}^{+}}{2}:=\sum_{n} \omega_{n}\left(a_{n}^{+} a_{n}^{-}+\frac{1}{2}\right)
$$

leads to the vacuum (Casimir) energy $\sum_{n} \frac{\omega_{n}}{2}$ [11]. The normal ordering of gluons $A^{a}$ in the SU(3) QCD including the product of covariant derivatives

$$
\nabla^{d b}(A) A_{0}^{b} \nabla^{d c}(A) A_{0}^{c}=: \nabla^{d b}(A) A_{0}^{b} \nabla^{d c}(A) A_{0}^{c}:+M_{g}^{2} A_{0}^{d} A_{0}^{d}
$$

leads to their effective gluon mass $M_{g}$ expressed in terms of the condensate $C_{\text {gluon }}$ :

$$
g^{2} f^{b a_{1} d} f^{d a_{2} c}\left\langle A_{i}^{a_{1}} A_{j}^{a_{2}}\right\rangle=3 g^{2}\left[N_{c}^{2}-1\right] \delta^{b c} \delta_{i j} C_{\text {gluon }}=M_{g}^{2} \delta^{b c} \delta_{i j},
$$

where $N_{c}=3$ and $g^{2}$ is the QCD coupling constant. It yields the Yukawa interaction in QCD

$$
V_{\mathrm{Yu}}(\mathbf{k})=\frac{4}{3} g^{2} \frac{1}{\mathbf{k}^{2}+M_{g}^{2}} .
$$

The ladder diagram sum of Coulomb interaction in QED leads to the Schrödinger equation. In the same way, in QCD, the ladder diagram sum of the Yukawa interaction between quarks leads to the Salpeter equation [3, 4] and the Schwinger-Dyson one for the mass operator $\Sigma(k)[12]$ :

$$
\Sigma(k)=m^{0}+i \int \frac{d q_{0} d^{3} q}{(2 \pi)^{4}} V_{\mathrm{Yu}}\left(k^{\perp}-q^{\perp}\right) \ell G_{\Sigma}(q) \ell,
$$

where $G_{\Sigma}(q)=(\not q-\Sigma(q))^{-1}, \not q \equiv q_{\mu} \gamma^{\mu}, k_{\mu}^{\perp}=k_{\mu}-\ell_{\mu}(k \cdot \ell), \ell^{2}=1, \ell \equiv \ell_{\mu} \gamma^{\mu}$, and $m^{0}$ is a current mass. In the reference frame $\ell^{0}=(1,0,0,0), q^{\perp}=(0, \mathbf{q})$ we can put

$$
\Sigma(q) \equiv M(\mathbf{q})=\sqrt{M^{2}+\mathbf{q}^{2}} \cos v(\mathbf{q}) \Rightarrow \cos v(\mathbf{q})=\frac{M(\mathbf{q})}{\sqrt{M^{2}+\mathbf{q}^{2}}}
$$

and take integration over $q_{0}$ in the S-D equation (2.6) for the constituent quark mass $M(\mathbf{p})$

$$
M(\mathbf{p})=m^{0}+\frac{1}{2} \int \frac{d^{3} q}{(2 \pi)^{3}} V(\mathbf{p}-\mathbf{q}) \cos v(\mathbf{q}) .
$$


In agreement with the Goldstone theorem, in the limit of small current masses of d- and u- quarks $m_{u} \simeq m_{d} \ll M_{g}$ and meson ones $M_{\pi} \rightarrow 0$ the Schwinger-Dyson equation (2.7) multiplied by a factor $\left(\sqrt{2} F_{\pi}\right)^{-1}$ coincides with the Salpeter equation

$$
\frac{M_{\pi} L_{(2)}^{\pi}(\mathbf{p})}{2}=\sqrt{\mathbf{p}^{2}+M_{d}^{2}(\mathbf{p})} L_{(1)}^{\pi}(\mathbf{p})-\frac{1}{2} \int \frac{d^{3} q}{(2 \pi)^{3}} V(\mathbf{p}-\mathbf{q}) L_{(1)}^{\pi}(\mathbf{q}),
$$

where $F_{\pi}=93 \mathrm{GeV}$ is the weak-decay coupling constant and

$$
\begin{aligned}
& L_{(1)}^{\pi}(\mathbf{p})=\frac{M_{d}(\mathbf{p})}{\sqrt{2} F_{\pi} \sqrt{\mathbf{p}^{2}+M_{d}^{2}(\mathbf{p})}}=\frac{\cos v_{d}(\mathbf{p})}{\sqrt{2} F_{\pi}}, \\
& L_{(2)}^{\pi}(\mathbf{p})=\frac{2 m_{d}}{\sqrt{2} F_{\pi} \cdot M_{\pi}}
\end{aligned}
$$

are the wave functions. In this case, the normalization condition

$$
4 N_{c} \int \frac{d^{3} q}{(2 \pi)^{3}} L_{(1)}^{\pi}(\mathbf{q}) L_{(2)}^{\pi}(\mathbf{q})=M_{\pi}
$$

yields the Gell-Mann-Oakes-Renner (GMOR) relation [13]

$$
M_{\pi}^{2} F_{\pi}^{2}=2 m_{d}<d \bar{d}>
$$

between the light quark condensate

$$
\langle d \bar{d}\rangle=\sum_{n=1}^{N_{c}}\left\langle q_{n}(t, \mathbf{x}) \bar{q}_{n}(t, \mathbf{x})\right\rangle=4 N_{c} \int \frac{d^{3} q}{(2 \pi)^{3}} \frac{1}{2} \cos v_{u}(\mathbf{q}),
$$

the current mass $m_{d}$, the pion mass $M_{\pi}$, and its weak decay coupling constant $F_{\pi}$.

In the chiral massless limit $\left(m^{0} \rightarrow 0\right)$ the solution of the Schwinger-Dyson equation was given in paper by Cherny et al. [14] in the form of a step-function. In the step-function approximation, it was shown in [9] that the Schwinger-Dyson equation and the Salpeter one yield meson spectrum via the constituent quark masses $M_{\text {const }} \simeq 330 \mathrm{GeV}$. Using the GMOR relation (2.11) and the constituent quark mass value $\sim 330 \mathrm{MeV}$ we can define a conformal invariant as the ratio of the condensate value to the cubed constituent mass

$$
\frac{<d \bar{d}>}{M_{d}^{3}}=\frac{M_{\pi}^{2} F_{\pi}^{2}}{2 m_{d} M_{d}^{3}} \simeq 0.41 \pm 0.08
$$

In the Dirac approach to QCD the color confinement means the complete destructive interference of phase factors $v^{(n)}(x)$ of the topological degeneration $(n)$ of the color states [15], so that the ordinary plane wave $e^{i p x}$ is replaced by the sum over parameters of these phase factors

$$
\sum_{n=-\infty}^{+\infty} v^{(n)}(x) \underbrace{e^{i p x}}_{\text {parton }}=0
$$

if $x \neq 0$. This means that amplitudes of the color particle creation are equal to zero. In this case, the left side of the optical theorem for $\hat{S}=1+i \hat{T}$

$$
\sum_{h}<*|\hat{T}| \underbrace{h><h}_{\text {hadrons }}|\hat{T}| *>=\underbrace{2 \operatorname{Im}<*\left|\hat{T}_{\text {Perturbation Theory }}\right| *>}_{\text {partons }} ;
$$

is the sum over only colorless hadron states; whereas the right side of the optical theorem is known as Feynman's parton model [15], where all colorless states are marked by stars. 


\section{Conformal Version of Minimal Standard Model}

Now, in the framework of the condensate mechanism of conformal symmetry breaking, we consider the conformal version of SM (CSM) [16]. For the beginning in the CSM Lagrangian one keeps only the scalar field potential part forming the four interaction and the largest mass t-quark Higgs field interaction

$$
L_{\mathrm{int}}=-\frac{\lambda^{2}}{8} h^{4}-g_{t} h \bar{t} t
$$

where $g_{t}=1 / \sqrt{2}$. Here, we consider only the most intensive terms: the self-interaction and the Yukawa ones of the top quark coupling constant $g_{t}$. The normal ordering of a fermion pair $f \bar{f}=$ : $f \bar{f}:+\langle f \bar{f}\rangle$ yields the condensate density of the fermion field $\langle f \bar{f}\rangle$ in the Yukawa interaction term in Eq.(3.1). The t-quark condensate as a consequence of the normal ordering

$$
V_{\text {cond }}(h)=\frac{\lambda^{2}}{8} h^{4}-g_{t}<t \bar{t}>h
$$

supersedes the phenomenological negative square mass term in the Higgs potential. The extremum condition for the potential $d V_{\text {cond }} /\left.d h\right|_{h=v}=0$ yields the relation

$$
v^{3} \frac{\lambda^{2}}{2}=g_{t}\langle t \bar{t}\rangle \text {. }
$$

This relation follows from the fact that the Higgs field has a zero harmonic $v$ in the standard decomposition of the field $h$ over harmonics $h=v+H$, where $H$ is the sum of all nonzero harmonics with a condition $\int d^{3} x H=0$. The substitution $h=v+H$ into the potential (3.2) leads to the result

$$
V_{\text {cond }}(h)=V_{\text {cond }}(v)+\frac{m_{H}^{2}}{2} H^{2}+\frac{\lambda^{2} v}{2} H^{3}+\frac{\lambda^{2}}{8} H^{4},
$$

which defines the scalar particle mass as

$$
m_{H}^{2}=\frac{3 g_{t}\langle t \bar{t}\rangle}{v} .
$$

The value of the t-quark condensate $\bar{t} t=: \bar{t} t:-\langle t \bar{t}>$ is estimated using the conformal invariant as the ratio of quark condensate and its cubed mass obtained above (2.12)

$$
\frac{<t \bar{t}>}{M_{t}^{3}}=\frac{<d \bar{d}>}{M_{d}^{3}} \simeq 0.41 \pm 0.08,
$$

where $M_{t}=173 \mathrm{GeV}$ is inputting parameter yielding the value of the constant part of the Higgs field $\mathrm{v}=246 \mathrm{GeV}$. In the tree approximation we obtain the value of the scalar particle mass

$$
m_{H}^{0}=130 \pm 15 \mathrm{GeV}
$$

Here, we have assigned $10 \%$ uncertainty into the ratio light quark condensate and its constituent mass. Contributions to this mass from other electroweak boson condensates $\left\langle W^{+} W^{-}\right\rangle$and $<Z Z>$ including the Higgs one $<H H>$ are very small [16]

$$
m_{H}=m_{H}^{0}\left[1+4 \frac{\Delta m_{H}^{2}}{v^{2}}\right]^{1 / 2} \approx m_{H}^{0} \cdot(1+0.02),
$$

in comparison with the accuracy of the constituent mass definition. 


\section{Dirac Conformal General Relativity and Empty Universe Model}

Instead of the Hilbert action $W_{\mathrm{H}}=-(1 / 6) \int d^{4} x \sqrt{-g} R^{(4)}$ and an interval $d s^{2}=g_{\mu \nu} d x^{\mu} d x^{\nu}$ in the Riemannian space-time in natural units: $M_{\mathrm{Pl}} \sqrt{3 /(8 \pi)}=c=\hbar=1$ we consider the Dirac action of the General Relativity $[2,17]$ with a scalar dilaton field $D$

$$
W_{\mathrm{CGR}}=-\int d^{4} x\left[\frac{\sqrt{-\widetilde{g}}}{6} R^{(4)}(\widetilde{g}) e^{-2 D}-e^{-D} \partial_{\mu}\left(\sqrt{-\widetilde{g}} \widetilde{g}^{\mu v} \partial_{v} e^{-D}\right)\right]
$$

and a long distance interval expressed through the Fock linear gauge-invariant forms $\widetilde{\omega}_{(\alpha)}^{\text {Fock }}$ [18]

$$
\widetilde{d s}^{2}=\widetilde{g}_{\mu v} d x^{\mu} d x^{v}=\widetilde{\omega}_{(\alpha)}^{\text {Fock }} \otimes \widetilde{\omega}_{(\beta)}^{\text {Fock }} \eta^{(\alpha)(\beta)},
$$

where $\eta^{(\alpha)(\beta)}$ is a local tangent Minkowskian space-time metrics. The frame of reference used for the $4=3+1$ foliation of the space-time manifold was given by Dirac [19] and Arnowitt-DeserMisner [20]:

$$
\widetilde{\omega}_{(0)}^{\text {Fock }}=e^{-2 D} N d x^{0}, \quad \widetilde{\omega}_{(b)}^{\text {Fock }}=\mathbf{e}_{(b) i} d x^{i}+N_{(b)} d x^{0} .
$$

Here $N$ is the lapse function presented as: $N=N_{0}\left(x^{0}\right) \mathscr{N}\left(x^{0}, x^{1}, x^{2}, x^{3}\right)$, where $V_{0}^{-1} \int_{V_{0}} d^{3} x \mathscr{N}^{-1}=1$, and $V_{0}=\int d^{3} x$ is the space volume; $N_{(b)}=N^{j} \mathbf{e}_{(b) j}$ are the shift vector components; $\mathbf{e}_{(b) i}$ are the space triad components with unit determinant.

The scalar dilaton field $D$ can be decomposed over the harmonics

$$
D\left(x^{0}, x^{1}, x^{2}, x^{3}\right)=\langle D\rangle\left(x^{0}\right)+\bar{D}\left(x^{0}, x^{1}, x^{2}, x^{3}\right),
$$

where $\langle D\rangle\left(x^{0}\right)$ is the dilaton zero mode and $\bar{D}\left(x^{0}, x^{1}, x^{2}, x^{3}\right)$ is the sum of other modes with the constraint $\int_{V_{0}} d^{3} x \bar{D}=0$. The dilaton zero mode plays the role of a reciprocal cosmological scale factor logarithm

$$
\langle D\rangle=-\ln a=\ln (1+z),
$$

where $z=(1-a) / a$ is the redshift.

Then, the action is split into the Newton-type part, graviton part and homogeneous one associated with the dynamics of the Universe as a whole:

$$
W_{\mathrm{CGR}}=W_{\text {potential }}+W_{\text {graviton }}^{\text {Fock }}+W_{\text {Universe }}
$$

The first part of the action (4.4)

$$
W_{\text {potential }}=\int d^{4} x N[-v_{\bar{D}}^{\frac{2}{3}}-\underbrace{\frac{4}{3} e^{-7 D / 2} \triangle^{(3)} e^{-D / 2}}_{\text {Newtonian potentials }}],
$$

yields the Newtonian potentials in the frame comoving to the local volume element velocity $v_{\bar{D}}=0$.

The second part of the action (4.4)

$$
W_{\text {graviton }}^{\text {Fock }}=\int d^{4} x \frac{N}{6}\left[v_{(a)(b)} v_{(a)(b)}-e^{-4 D} R^{(3)}(\mathbf{e})\right]
$$


describes the one-component graviton in a squeezed state [17], where the parameter of squeezing is the dilaton. In the Beginning, according to the Bible (Genesis 1:2), the Universe was empty. From physical point of view this means that the second and third parts of the action (4.4) were equal to zero:

$$
W_{\text {graviton }}=W_{\text {potential }}=0 .
$$

The third part of the action (4.4) describes the zero mode dynamics of the Universe as a whole:

$$
W_{\text {Universe }}=-V_{0} \int_{\tau_{I}}^{\tau_{0}} \underbrace{d x^{0} N_{0}}_{=d \tau}\left[\left(\frac{d\langle D\rangle}{N_{0} d x^{0}}\right)^{2}+\rho_{\text {Cas }}^{\tau}\right] \text {. }
$$

Here $d \tau=N_{0}\left(x^{0}\right) d x^{0}$ is a luminosity time interval connected with the conformal time interval $d \eta$ and the world one $d t$ by the relations from (4.1) and (4.2):

$$
d \tau=a^{-2} d \eta=a^{-3} d t
$$

$\rho_{\text {Cas }}^{\tau}$ is the Casimir vacuum energy density corresponding to the luminosity time interval $\tau$.

In the conformal units the Casimir energy density is able to be represented as a sum over all physical field energies $\mathrm{H}_{\text {Cas }}^{(f)}$ :

$$
\rho_{\mathrm{Cas}}^{\eta}(a)=\frac{\rho_{\mathrm{Cas}}^{\tau}}{a^{2}}=\sum_{f} \frac{\mathrm{H}_{\mathrm{Cas}}^{(f)}}{V_{0}}=\frac{H_{0}}{d_{\mathrm{Cas}}(a)},
$$

where $d_{\text {Cas }}(a)$ is the conformal size of the Universe and $H_{0}$ is the Hubble parameter.

Variations of the action with respect to two independent variables $\langle D\rangle$ and $N_{0}$ give the equations of the Empty Universe

$$
\begin{aligned}
& \frac{\delta W_{\text {Universe }}}{\delta\langle D\rangle}=0 \Rightarrow 2 \frac{d}{d \tau}\left[\frac{d\langle D\rangle}{d \tau}\right]=\frac{d \rho_{\mathrm{Cas}}^{\tau}}{d\langle D\rangle}, \\
& \frac{\delta W_{\text {Universe }}}{\delta N_{0}}=0 \Rightarrow\left[\frac{d\langle D\rangle}{d \tau}\right]^{2}=\rho_{\text {Cas }}^{\tau} .
\end{aligned}
$$

The latter equation rewritten in terms of the conformal cosmological factor $a=\exp (-\langle D\rangle)$ and the conformal density $\rho_{\text {Cas }}^{\eta}(a)$ coincides with the Friedmann equation

$$
\left[\frac{d a}{d \eta}\right]^{2}=\rho_{\mathrm{Cas}}^{\eta}(a) .
$$

Solution of the Friedmann equation (4.10) yields the conformal horizon

$$
d_{\text {horison }}(a)=2 r_{\text {horison }}(a)=2 \int_{0}^{a} d \bar{a}\left[\rho_{\text {Cas }}^{\eta}(\bar{a})\right]^{-1 / 2} .
$$

The horizon is defined as the distance that a photon covers within its light cone $d \eta^{2}-d r^{2}=0$ for life-time of the Universe. In our case, the horizon coincides with the visual size of the Universe $d_{\text {Cas }}(a)$ in (4.7):

$$
d_{\text {Cas }}(a)=d_{\text {horison }}(a)
$$


The solution of equations (4.3), (4.7), (4.11), and (4.12)

$$
d_{\text {horison }}(a)=\frac{a^{2}}{H_{0}} \Rightarrow \rho_{\text {Cas }}^{\tau}=H_{0}^{2} \equiv \rho_{\text {cr }}
$$

yields the Hubble diagram of the description of Supernova Data in Conformal Cosmology [21, 22] obtained as a consequence of the Dirac conformal GR in the void space approximation. In Figure 1 you can see that the conformal long space interval $R_{\text {long }}=r$ explains long Supernovae distances via the dominant Casimir energy (see black line) without the cosmological constant in the framework of the Empty Universe Model given by (4.5) and (4.12).

While the Lambda Cold Dark Matter standard model with a short space interval $R_{\text {short }}=r a$ requires the $\Lambda$ term dominance to explain the long Supernovae Distances.

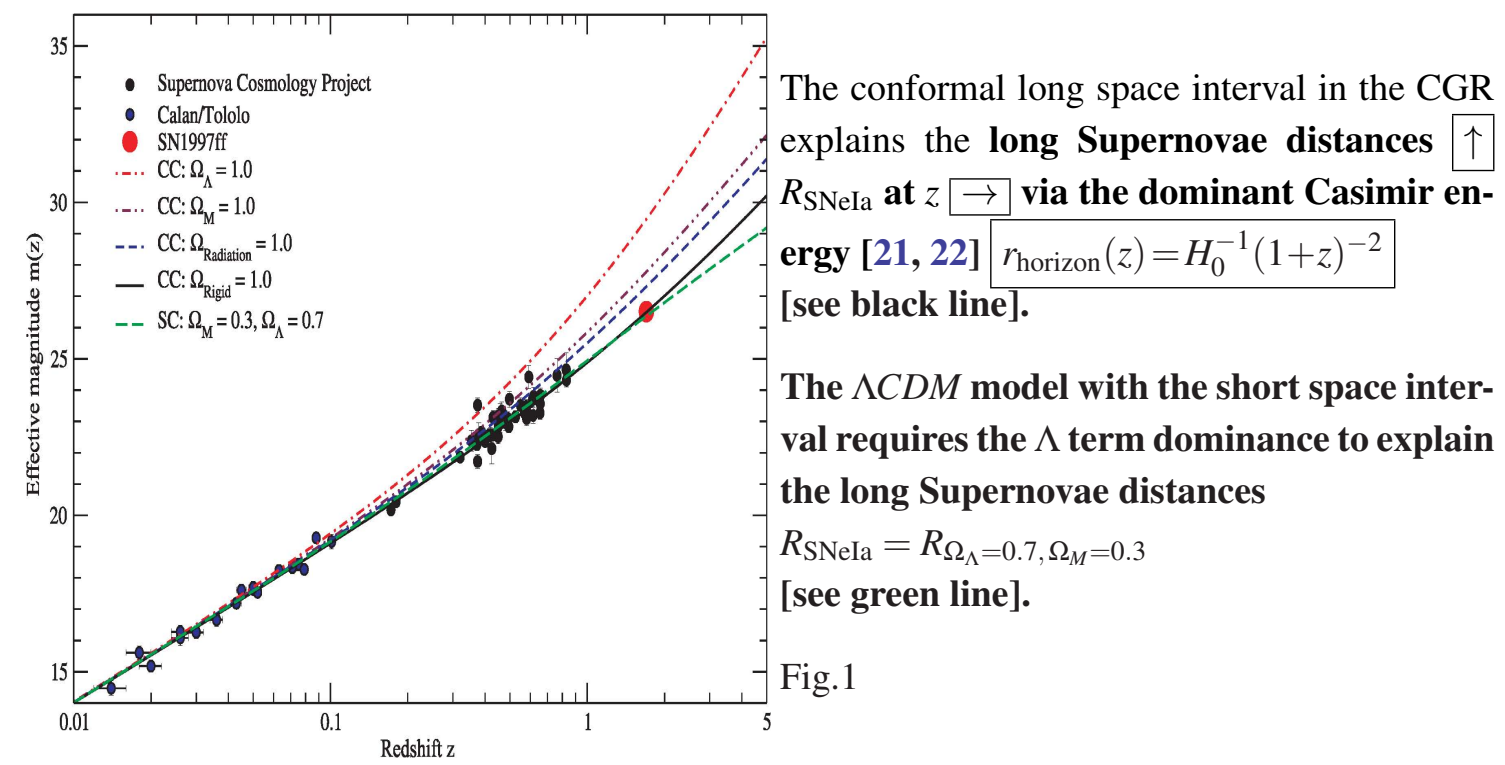

In paper [2], the Empty Universe Model was presented in detail. It was demonstrated that the Planck least action postulate applied to the Universe limited by its horizon yields the value of the cosmological scale factor in the Planck epoch. In other words, the Planck least action postulate

$$
W_{\text {Universe }}=\rho_{\mathrm{cr}} V_{\text {hor }}^{(4)}\left(a_{\mathrm{Pl}}\right)=M_{\mathrm{Pl}}^{2} H_{0}^{2} \frac{1}{H_{0}^{4}} \frac{\left(1+z_{\mathrm{Pl}}\right)^{-8}}{32}=2 \pi
$$

yields the redshift at the Beginning: $a_{\mathrm{Pl}}^{-1}=\left(1+z_{\mathrm{Pl}}\right) \simeq 0.85 \cdot 10^{15}$. One can see that in the Empty Universe Model the Planck epoch coincides with the electro-weak one.

One can consider, in the tangent space-time, representations of the Weyl group [23] which includes besides of the Poincaré group the scale transformations. This means that a massive particle energy with respect to the luminosity time interval: $d \tau=d \eta / a^{2}$ takes the form

$$
\omega_{\tau}(a)=a^{2} \sqrt{\mathbf{k}^{2}+a^{2} M_{0}^{2}}
$$

This energy can be decomposed into different conformal weight parts

$$
<\omega_{\tau}^{(\mathrm{n})}(a)>=\frac{a^{n}}{a_{\mathrm{Pl}}^{n}} H_{0}
$$


responsible for the representations of the Weyl group in the tangent Minkowskian space-time. These representations give scales

$$
<\omega_{\tau}^{(\mathrm{n})}(a)>\left.\right|_{a=1}=\frac{H_{0}}{a_{\mathrm{Pl}}^{n}}
$$

for conformal weights $\mathrm{n}=0,1,2,3,4$ in $\mathrm{GeV}$ :

\begin{tabular}{|c|c|c|c|c|}
\hline $\mathrm{n}=0$ & $\mathrm{n}=1$ & $\mathrm{n}=2$ & $\mathrm{n}=3$ & $\mathrm{n}=4$ \\
\hline$H_{0} \sim 10^{-42}$ & $R_{\text {Celestial system }}^{-1} \sim 10^{-27}$ & $T_{\mathrm{CMB}} \sim 10^{-12}$ & $M_{\mathrm{EW}} \sim 10^{3}$ & $M_{\mathrm{PI}} \sqrt{3 /(8 \pi)} \sim 10^{18}$ \\
\hline
\end{tabular}

Thus, conformal weights of the Weyl group representations with respect to the luminosity energies give us the present-day $(a=1)$ mass scales of an order of the electroweak scale energy for $n=3$, a photon energy for $n=2$ of an order of the CMB temperature, and nonrelativistic energy $\omega_{\tau}^{(1)}(a)=a k^{2} /\left(2 M_{0}\right)$ for $n=1$ of an order of the Celestial system inverse size. The Weyl group representations leads to the classification of energy scales that points out the common origin of conformal symmetry breaking in both GR and SM.

The intensive creation of primordial gravitons and Higgs bosons is described assuming that the Casimir vacuum energy is the source of this process [2].

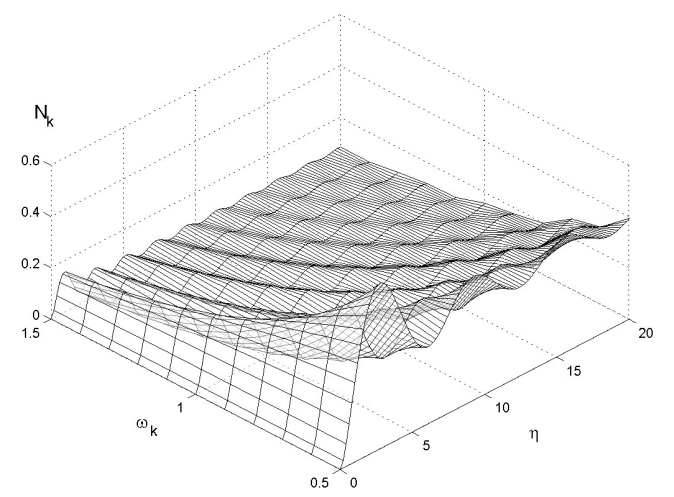

The Casimir energy is the source of creation of $10^{90}$ Higgs particles [2]. This Figure, where $\nearrow$ is time-axis,

$\uparrow$ is number of bosons $N_{W, Z, h}$,

$\searrow$ is their momentum, shows us creation of $N_{h} \sim 10^{90}$ Higgs particles at $1+z_{\mathrm{Pl}} \sim 10^{15}$ during the first $10^{-12}$ sec.

Fig. 2.

The Casimir energy is the source of creation of $10^{90}$ Higgs particles that decay into $10^{87}$ photons with $\mathrm{CMB}$ temperature $\sim 3 \mathrm{~K}$ in the conformal units.

\section{Conclusion}

At the present talk we tried to demonstrate the mysterious and intrigue fruitfulness of the conformal symmetry applications: its "footprints" manifest themself on the whole accessible scale - from quarks to horizons of the Universe. For illustration we superinduce explicitness in some problems taken from various fields of theoretical physics: application of the low energy GellMann-Oakes-Renner relation to QCD, appearance of the Higgs particle mass in Minimal Standard Model of electroweak interactions without having used the Higgs potential, interpretations of new observational cosmological data in framework of the Conformal GR. Everywhere the conformal symmetry breaking is manifested via the condensate mechanism at the quantum level of description of phenomena. 


\section{References}

[1] P.A.M. Dirac, Proc. Roy. Soc. Lond. A 333 (1973) 403.

[2] V.N. Pervushin, A.B. Arbuzov, B.M. Barbashov, R.G. Nazmitdinov, A. Borowiec, K.N. Pichugin, and A.F. Zakharov, Gen. Relativ. Gravit., 44 (2012) 2745.

[3] E.E. Salpeter and H.A. Bethe, Phys. Rev. 84 (1951) 1232.

[4] E.E. Salpeter, Phys. Rev. D 87 (1952) 328.

[5] M.A. Markov, J. Phys. (USSR) 3 (1940) 453; H. Yukawa, Phys. Rev. 77 (1950) 219.

[6] V.N. Pervushin, Nucl. Phys. B (Proc. Supp.) 15 (1990) 197.

[7] P.A.M. Dirac, Proc. Roy. Soc. A 114 (1927) 243; Can. J. Phys. 33 (1955) 650.

[8] N.N. Bogoliubov, A.A. Logunov, A.I. Oksak, and I.T. Todorov, General Principles of Quantum Field Theory, Springer (1989).

[9] Yu.L. Kalinovsky, L. Kaschluhn, and V.N. Pervushin, Phys. Lett. B 231 (1989) 288; Fortsch. Phys. 38 (1990) 353.

[10] W. Heisenberg and W. Pauli, Z. Phys. 56 (1929) 1; Z. Phys. 59 (1930) 166.

[11] A.A. Actor, Fortsch. Phys. 43 (1995) 141;

M. Bordag, G.L. Klimchitskaya, U. Mohideen, and V.M. Mostepanenko, Advances in the Casimir Effect, Oxford University Press Inc., New York (2009).

[12] F. Dyson, Phys. Rev., 75 (1949) 1736; J. Schwinger, PNAS 37 (1951) 452.

[13] M. Gell-Mann, R.J. Oakes, and B. Renner, Phys. Rev. 175 (1968) 2195.

[14] A.Yu. Cherny, A.E. Dorokhov, Nguyen Suan Han, V.N. Pervushin, V.I. Shilin, Bound States in Gauge Theories as the Poincaré Group Representations, arXiv:1112.5856 [hep-th].

[15] V. Pervushin, Riv. del Nuovo Cimento 8 (1985) N 10, 1-48.

[16] V.N. Pervushin, A.B. Arbuzov, R.G. Nazmitdinov, A.E. Pavlov, A.F. Zakharov, Condensate Mechanism of Conformal Symmetry Breaking and the Higgs Boson, arXiv:1209.4460 [hep-ph].

[17] A.B. Arbuzov, B.M. Barbashov, R.G. Nazmitdinov, V.N. Pervushin, A. Borowiec, K.N. Pichugin, A.F. Zakharov, Phys. Lett. B 691 (2010) 230; [arXiv:1007.0293 [gr-qc]].

[18] V. Fock, Z. Phys. 57 (1929) 261.

[19] P.A.M. Dirac, Proc. Roy. Soc. Lond. A 246 (1958) 333.

[20] R. Arnowitt, S. Deser, and C.W. Misner, The dynamics of general relativity, in L. Witten, Gravitation: An Introduction to Current Research, Wiley, New York (1962) p.227.

[21] D. Behnke, D.B. Blaschke, V.N. Pervushin and D. Proskurin, Phys. Lett. B 530 (2002) 20; [arXiv:gr-qc/0102039].

[22] A.F. Zakharov and V.N. Pervushin, Int. J. Mod. Phys. D 19 (2010) 1875; [arXiv:1006.4745 [gr-qc]]; A.F. Zakharov, V.N. Pervushin, Physics of Atomic Nuclei, 75 (2012) 1418.

[23] P. Ramond, Field Theory. A Modern Primer, The Benjamin / Cummings Publishing Company, Inc. (1981). 